\title{
Coupled Fusion Simulation Using the Common Component Architecture ${ }^{\star}$
}

\author{
Wael R. Elwasif ${ }^{1}$, Donald B. Batchelor ${ }^{2}$, David E. Bernholdt ${ }^{1}$, Lee A. Berry ${ }^{2}$, \\ Ed F. D’ Azevedo ${ }^{1}$, Wayne A. Houlberg ${ }^{2}$, E.F. Jaeger ${ }^{2}$, James A. Kohl ${ }^{1}$, \\ and Shuhui $\mathrm{Li}^{3}$ \\ 1 Computer Science and Mathematics Division \\ 2 Fusion Energy Division \\ Oak Ridge National Laboratory, Oak Ridge, TN 37831 USA \\ \{elwasifwr, batchelordb, bernholdtde, berryla, dazevedoef, \\ houlbergwa, jaegeref, and kohlja\}@ornl.gov \\ 3 Department of Electrical Engineering \& Computer Science, Texas A\&M \\ University-Kingsville, MSC 192, Kingsville, TX 78363 USA \\ Shuhui.Li@tamuk. edu
}

\begin{abstract}
The physics of magnetically-confined fusion plasmas involves many different processes with multiple time and length scales that cover many orders of magnitude. As the capability of large parallel computers continues to grow, the goal of an integrated self-consistent simulation of all of the relevant physics draws closer. However, advances in computer science, physics formulations, and algorithms are also needed to achieve this goal. In this paper, we present an overview of an on-going project which is exploring these issues in the context of integrated simulation of radio frequency (RF) heating and transport physics as an initial step toward whole-device modeling. We present our experience in using the common componment architecture (CCA) as the underlying framework for the integration of the different physics modules. This work illustrates the viability of using high performance component technology in a complex simulation environment.
\end{abstract}

\section{Introduction}

The processes involved in modelling magnetically-confined fusion plasmas include electromagnetic-wave plasma interactions, transport (both collisional and turbulent) particle drift orbit effects, magnetohydrodynamics, atomic physics, and plasma-wall interactions. Research groups have developed codes to model these processes individually, or in a very loosely coupled fashion (generally without self-consistency). However scientific needs for planned systems, such as the International Thermonuclear Experimental Reactor (ITER) project $^{1}$, require the capability to model entire devices in an integrated, self-consistent fashion. This coupling requires that the output of one simulation process provides (time varying) boundary conditions or inputs to the other physical

\footnotetext{
${ }^{\star}$ Research sponsored by the Laboratory Directed Research and Development Program of Oak Ridge National Laboratory (ORNL), managed by UT-Battelle, LLC for the U. S. Department of Energy under Contract No. DE-AC05-00OR22725.

${ }^{1}$ http://www.iter.org/
} 
process model simulations. A complete, integrated whole device model (WDM) simulation would encompass time scales ranging from nanoseconds to tens of minutes and length scales from the subatomic to the size of the reactor vessel itself ( $\sim 6$ meters tall).

The growing capability of large parallel computers continually increases the feasibility of this type of integrated simulation, at least in terms of the raw computational power required. However, significant challenges still must be overcome to make largescale coupled simulations, such as WDM, a practical reality. Advances must be made in computer science, algorithms and in the physics formulations themselves [1]. For example, while a well-tested physical model will typically provide satisfactory results in its original (uncoupled or loosely coupled) context, the behavior may become more complex and harder to understand when additional physical processes are coupled in because assumptions under which the original model was developed may break down. Likewise, the algorithms and mathematical approaches used in the individual models must often change to accommodate the new coupled interactions. For example, when coupling two simulations where the natural time step differs by a factor of a million, it is often not feasible to actually run a million steps of the fast model between successive steps of the slower one, as a naive integration approach might attempt. Alternative algorithms and solution strategies are required to preserve the essential physics while accelerating the coupled solution to a more practical level. In addition, the integration of codes developed by disparate groups raises software-related issues ranging from reconciling software architectures and facilitating software-based collaboration, to the detailed mechanics of integrating software that may have been developed using different parallel programming models, written in different incompatible languages, and use different data structures.

In order to examine these issues in more detail and lay the groundwork for largerscale efforts to develop a comprehensive integration fusion simulation capability [1], we have undertaken a modest "prototype" integrated fusion simulation effort. Our project focuses initially on the coupling of just two physical processes: RF heating of the plasma, which occurs on the nanosecond time scale, with transport within the plasma, which occurs on the millisecond time scale. We have developed an "evolutionary preconditioning" approach to address the disparity in time scales between these two models, and have used the Common Component Architecture (CCA $)^{2}$ as an efficient and flexible framework in which to integrate the independent simulation codes. In this paper, we present a high-level overview of our ongoing effort, emphasizing the computer science and mathematical aspects of the project.

This paper is organized as follows: in Section 2 we present, briefly, a description of the RF heating and transport processes and how they are simulated; Section 3 describes the evolutionary preconditioning approach used to numerically facilitate the integration; and, Section 4 introduces the Common Component Architecture and its utility for this work. Then in Section 5 we describe our prototype integrated simulation application and share our experiences developing it. We present our Conclusions and plans for Future Work in Section 6.

\footnotetext{
${ }^{2}$ http://www.cca-forum.org
} 


\section{Plasma Physics: RF Heating and Transport}

Plasma transport, typically particle convection; current and particle diffusion; and thermal conduction, combined with the boundary conditions and sources for the fluxes, control the evolution of macroscopic plasma parameters. The transport coefficients used to model the fluxes can be highly non-linear and, in some cases, non-local in space and time. As a result, the time and space dependence of the temperatures, densities, and magnetic fields that comprise the desired output can be quite complex. Time scales for these processes are in the range of hundreds of milliseconds to tens of seconds for present and planned experiments.

The transport of particles, heat, and current (or equivalently magnetic flux) are each described by a partial differential equation. For example, heat transport for a particular plasma species $i$ is described by an equation of the form

$$
\frac{\partial\left(n_{i} T_{i}\right)}{\partial t}+\vec{\nabla} \cdot K_{i} \vec{\nabla}\left(n_{i} T_{i}\right)=S_{i}
$$

where $n_{i}$ is the density of species $i, T_{i}$ is the temperature, $K_{i}$ is the thermal conductivity, and $S_{i}$ is the external source/sink of energy. For simplicity, convective terms and the interchange of energy between species due to friction and thermal equilibration have been omitted. Although the plasma is either 2-D or 3-D in space, systems with good confinement have magnetic field lines that form closed, nested surfaces. Because transport along field lines is much faster than that across field likes, we average the transport equations along magnetic field lines and solves for transport across these surfaces. Standard conservative finite different techniques and operator splitting are used for solving the coupled systems. Evolving the transport equations is much less computationally intensive than solving for the interactions of the plasma with RF waves.

Radio frequency (RF) power in the ion cyclotron frequency range $(\sim 50-200 \mathrm{MHz}$ at 10s of MW) is a principle means of plasma heating. Plasma parameters may be assumed constant for the tens of nanoseconds time scale for variation of the RF electromagnetic fields. Thus the waves reach steady state on the electromagnetic time scale before plasma parameters change significantly. Thus wave properties, for example energy deposition, may be calculated with stationary plasma parameters and subsequently used to advance the transport.

For many problems of interest, plasma responses to the applied RF power may be linearized in RF fields. Under these conditions, the All-Orders Spectral Algorithm (AORSA) solves for the wave fields needed for the plasma simulation [2] This code accurately treats the physics of waves that interact with arbitrary harmonics of the ion cyclotron frequency and with perpendicular wavelengths that are smaller than ion gyroradii in the strong equilibrium magnetic field. However, resolving the spatial dependence of the dominant wave-plasma interactions in 2-D requires the solution of from 10,000 to over 100,000 dense linear equations and can use over 10,000 processor hours. As a result, time-dependent plasma simulations were prohibitively expensive as hundreds of AORSA solutions might be required to resolve a few plasma energy confinement times. To address this issue, we have developed an "evolutionary preconditioning" approach to accelerate the coupled simulation. 


\section{Mathematics: Evolutionary Preconditioning}

One of the most time consuming computational kernels in the AORSA code is the solution of a large dense complex linear system by LU factorization in parallel using ScaLAPACK [3]. The matrix is highly non-symmetric and the spectrum has large components in the imaginary plane. This presents a significant challenge to most Krylov iterative solvers such as GMRES [4] and an effective preconditioner is required.

The key to implementing a time dependent simulation was the observation that the plasma parameters (determined by the transport simulation) are changing on a longer time scale, so we need results from a sequence of closely related RF calculations rather than independent solutions to a large number of independent simulations. Thus we use an "evolutionary" approach, using the exact LU decomposition for a set of plasma conditions at one time to precondition the AORSA linear equations that are determined by the parameters for a subsequent time step. Reusing the LU factorization from previous time steps as a preconditioner for an iterative solver offers the possiblity of reducing the $O\left(N^{3}\right)$ cost for factorization to $O\left(N^{2}\right)$ cost in triangular solves and matrix multiplies. When the plasma parameters have evolved sufficiently that the iterative method is no longer effective, a new direct solution will be recomputed. Hopefully, the need for such direct factorization would be limited to a sufficient small number of intermediate points to make the overall simulation practical.

This approach has been tested and proven effective over a reasonable range of plasma parameters. For "sensitive" parameters including electron density and magnetic field strengths, parameters could be varied $\pm 20 \%$ and still converge within 100 itera-

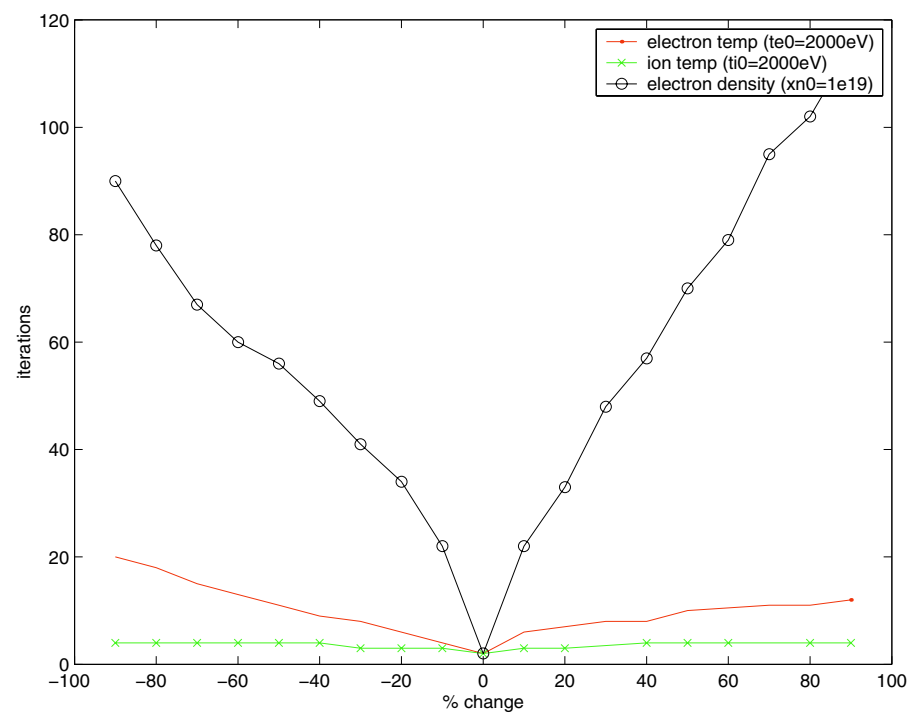

Fig. 1. Sensitivity of convergence of evolutionary preconditioning scheme to changes in various simulation parameters. Tests were done for a $32 \times 32$ mode problem, with a reduced matrix size $(N)$ of 1965 complex equations 
tions. While for "non-sensitive" parameters such as electron and ion temperatures and toroidal mode number of RF antenna, a variation of $\pm 50 \%$ was possible while maintaining good convergence. Fig. 1 illustrates the convergence characteristics of several simulation parameters. These results suggest that a significant number of transport time steps can be taken before a new preconditioner must be generated. We are currently working on incorporating this approach into the integrated simulation.

\section{Computer Science: The Common Component Architecture}

The computer science and software engineering aspects of building a coupled simulation are often at least as complex as the physics and mathematics involved. Issues range from the software (and social) engineering of getting disparate groups of software developers to collaborate around the integrated simulation to the details of reconciling different software architectures, programming styles, parallel programming models, programming languages, data structures, etc. In this work, we look to ideas of component-based software engineering (CBSE), and in particular the realization of those ideas in the Common Component Architecture (CCA) [5,6] to provide a software environment in which to integrate the coupled simulation and facilitate the social aspects of collaborative development.

CBSE is, fundamentally, a means to help manage the complexity inherent in modern large-scale software systems. CBSE treats applications as assemblies of software components that interact with each other only through well-defined interfaces (or ports in CCA terminology) within a particular execution environment, or framework. Components are units of software functionality, and are a logical means of encapsulating knowledge from one scientific domain (or sub-domain) for use by those in others, thereby facilitating group and multidisciplinary interactions. The glue that binds the components together is a set of common, agreed-upon interfaces, or ports. Multiple component implementations conforming to the same external interface standard should be interoperable, while providing flexibility to accommodate different aspects such as algorithms, performance characteristics, and coding styles. At the same time, the use of common interfaces facilitates the reuse of components across multiple applications. The Common Component Architecture distinguishes between provides ports, which are implemented by a component, and uses ports, where a component makes calls on a port provided by another component. The framework is responsible for providing an environment in which applications can be assembled from components (by connecting matching uses and provides ports) while insuring that internals of the components remain opaque to each other.

The CCA is designed specifically for the needs of parallel, scientific highperformance computing (HPC) in response to limitations of other, more widely used component approaches (see [5]). The general-purpose design of the CCA is intended for use in a wide range of scientific domains, and CCA-based simulations are under development in numerous fields, e.g. [7]. The CCA's emphasis on supporting HPC scientific computing has led to a number of important characteristics being incorporated into its design, and into tools that implement the CCA environment. 
The CCA's uses/provides design pattern allows components in the same process address space to be invoked directly, without intervention by the framework, and with data passed by reference if desired, preserving high performance for components which are local to each other [8]. In parallel computing, interactions among components within the same process are handled by the CCA's port-based mechanisms, taking advantage of the "direct connect" approach just mentioned. Interactions across parallel instances of a component are up to the component's developer, and carry no CCA-imposed overheads.

Language interoperability is an important feature of many component models because it allows components to be opaque with respect to implementation language as well as other implementation details. In the CCA, the Scientific Interface Definition Language (SIDL) [9] provides a means to express the interfaces between components independent of their implementation language. SIDL works in conjunction with the Babel language interoperability tool ${ }^{3}$ [9], which currently supports $\mathrm{C}, \mathrm{C}++$, Fortran 77, Fortran 90/95, Python, and Java.

Finally, the CCA employs a minimalist design philosophy to simplify the task of incorporating existing software into the CCA environment. The amount of extra code needed to acclimate a software module to the CCA environment can be as little as one extra (typically short) method, and several additional calls that manage ports provisioning and use. Experience has shown that componentization of existing software in the CCA environment is straightforward when starting from well-organized code [10].

\section{Prototype Integrated Fusion Simulation}

Our prototype integrated simulation was designed with the idea it should be extensible to integrate other physical processes in addition to the RF heating and transport simulations. In addition, we envisioned a simple, evolutionary migration path from the existing physics software to the componentized integrated simulation. The resulting design includes four types of components: a driver, which orchestrates the coupled simulation; the "physics" components, which encapsulate the original standalone simulation codes, an in-memory "plasma state" component, which holds the data exchanged by the different physics components, and "transformers" which adapt data from the form stored in the plasma state and the forms required by the various physics components. Fig. 2 shows the visual representation of the assembled application provided by the Ccaffeine CCA framework ${ }^{4}$.

While the physics components and the transformers are all written in Fortran 90, the driver is written in the Python scripting language to facilitate flexible and rapid experimentation with different driver algorithms and parameters, and the plasma state component is implemented in $\mathrm{C}++$.

The original standalone physics components read and write files directly for their inputs and outputs, and the initial version of the coupled application retains this characteristic (temporarily), using same file formats to transfer data between the plasma state

\footnotetext{
${ }^{3}$ http://www.llnl.gov/CASC/components/babel.html

${ }^{4} \mathrm{http}: / /$ www.cca-forum.org/ccafe/
} 


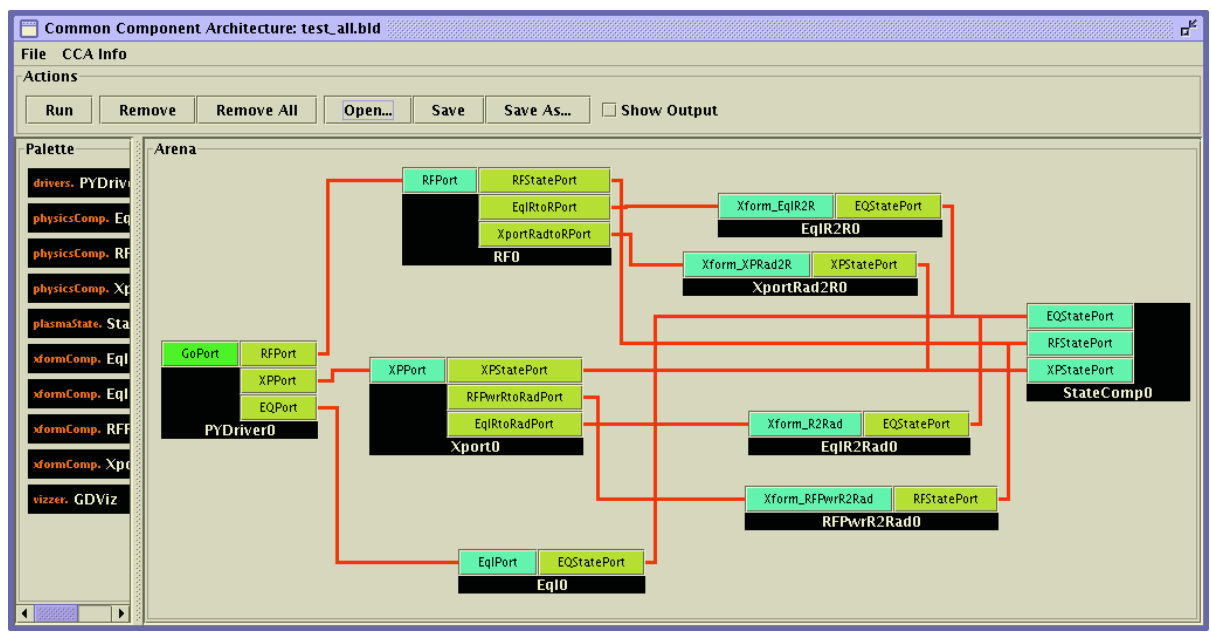

Fig. 2. Plasma simulation components

and the physics components. This approach was taken in order to minimize changes to the physics codes before verifying their correct behavior within the coupled application. We expect to quickly evolve the interfaces to allow for direct data exchange between components as the following step in the evolution of the port interfaces.

Data exchange between different physics module poses another challenge related to the format of the data. The output of one component may need to be re-gridded or transformed into a different coordinate system for another component. Most standalone physics codes in this community have their own set of transformation routines to perform these functions on inputs derived from other codes. In our initial prototype, we decided that the plasma state component should store data in the format in which it was computed. We use transformer components (extracted from the original standalone physics simulations) to implement a "receiver makes right" approach to insure components using the data get it in the format they need. While this approach does not address the $n^{2}$ problem of data transformation, we plan on using this prototype to gain a better understanding of the data transform issues in fusion simulations and develop general purpose reusable components that address this issue, drawing on existing efforts such as $[11,12]$.

The design of the interfaces that define the boundaries between the various components is crucial for the long-term flexibility and extensibility of the model. In our prototype, we opted for an evolutionary approach that facilitates rapid code integration, while allowing for future refinement as we gain better understanding of the overall application. A decision was made to adopt an initial design that promoted a loose component coupling, where methods that make up port interfaces are kept at a decidedly high level. This decision allows prototyping to proceed with little or no change to the mature physics applications that underly the main physics components. An added benefit of this approach has been the reduction in the amount of testing that had to be performed to validate the correctness of the overall application. 


\section{Conclusions and Future Work}

In this paper, we presented the physical, mathematical, and computer science issues involved in the design and implementation of a coupled fusion simulation using the Common Component Architecture. The prototype we developed couples together several physics modules, along with the necessary data and driver components. Our experience has demonstrated the viability of using CCA as the underlying foundation for such integration efforts. As we continue, our primary focus is on gaining a better understanding of the detailed needs of large-scale coupled fusion simulations and evolving our prototype to meet them. This includes, for example, expanding the capabilities of the prototype by introducing application controlled checkpointing and archival storage into the plasma state component. Another aspect of our future plan involves the refinement of the interfaces, with the ultimate goal of helping to develop interfaces that can be agreed to and used across the larger fusion community; this would be an important step towards coupled simulations integrating more physical processes.

\section{References}

1. Dahlburg, J., et al.: Fusion simulation project: Integrated simulation and optimization of fusion systems. J. Fusion Energy 20 (2001) 135-196

2. Jaeger, E., et al.: All-orders spectral calculation of radio-frequency heating in twodimensional toroidal plasmas. Physics of Plasmas 8 (2001) 1573-1583

3. Blackford, L.S., et al.: ScaLAPACK Users' Guide. SIAM (1997) Online version at http://www.netlib.org/scalapack/slug/index.html.

4. Saad, Y., Schultz, M.: GMRES: A generalized minimal residual algorithm for solving nonsymmetric linear systems. SIAM J. Sci. Statit. Comput. 7 (1986) 856-869

5. Bernholdt, D.E., et al.: A component architecture for high-performance scientific computing (2004) submitted to Intl. J. High-Perf. Computing Appl.

6. Armstrong, R., et al.: Toward a Common Component Architecture for high-performance scientific computing. In: Proceedings of the Eighth IEEE International Symposium on High Performance Distributed Computing. (1999)

7. McInnes, L.C., et al.: Parallel pde-based simulations using the common component architecture. In Bruaset, A.M., Bjørstad, P., Tveito, A., eds.: Numerical Solution of PDEs on Parallel Computers. Springer-Verlag (2005) invited chapter, accepted.

8. Bernholdt, D.E., Elwasif, W.R., Kohl, J.A., Epperly, T.G.W.: A component architecture for high-performance computing. In: Proceedings of the Workshop on Performance Optimization via High-Level Languages and Libraries (POHLL-02). (2002)

9. Dahlgren, T., Epperly, T., Kumfert, G.: Babel User's Guide. CASC, Lawrence Livermore National Laboratory. (2004) http://www.llnl.gov/CASC/components/babel.html.

10. Norris, B., et al.: Parallel components for PDEs and optimization: Some issues and experiences. Parallel Computing 28 (2002) 1811-1831

11. Bertrand, F., Bramley, R., Damevski, K.B., Kohl, J.A., Bernholdt, D.E., Larson, J.W., Sussman, A.: Data redistribution and remote method invocation in parallel component architectures. In: Proceedings of the 19th International Parallel and Distributed Processing Symposium: IPDPS 2005. (2005) submitted.

12. Glimm, J., Brown, D., Freitag, L.: Terascale Simulation Tools and Technologies (TSTT) Center. http://www.tstt-scidac.org (2001) 